\title{
Mathematical model of the cracking process in the coal-rock massif under hydrodynamic impact
}

\author{
Vasyl Vlasenko ${ }^{1, *}$, Kateryna Dudlia ${ }^{1}$, and Maryna Kyrychenko ${ }^{2}$ \\ ${ }^{1}$ Institute of Geotechnical Mechanics named by N. Poljakov of National Academy of Sciences of \\ Ukraine, 49005, Dnipro, Simferopolska Str., 2a, Ukraine \\ ${ }^{2}$ National Technical University Dnipro Polytechnic, 49005, Dnipro, Dmytra Yavornytskoho Ave., 19, \\ Ukraine
}

\begin{abstract}
The physics of hydrodynamic impact (HDI) is in the softening of the coal seam due to the generation of alternation stress in it. Stress is carried out due to injection and discharge of fluid into the coal seam in the filtration mode. The mathematical model of the processes occurring in the coal massif under HDI was developed. The model allows to determine the nature of the coal destruction in forward and inverse filtering of fluid in the massif. Cracking in the process of fluid filtration is achieved while simultaneously fulfilling two criteria: power and energy.
\end{abstract}

\section{Introduction}

The general formulation of the HDI problem on a porous medium it is solving a filtration equation with boundary conditions varying at certain points in time. This occurs during decompression and cleft of the porous medium. Therefore, the use of analytical solutions, divide the task into several subtasks: forward filtering, inverse filtering for the full solution region under consideration and inverse filtering problems for subdomains arising from the destruction of a material. This approach is too cumbersome and, considering the nonlinearity of the filtering equation, is practically unrealistic to implement. The only way out of this situation can be the use of numerical methods for solving partial differential equations. The most widely used difference methods based on the replacement of derivatives by finite differences. The theory of constructing difference schemes for equations of various types and equations systems is described in detail in the paper [1]. The general formulation of the mathematical problem is formulated.

Filtering equation

$$
\frac{\partial P}{\partial t}=H(P) \frac{1}{r^{n}} \frac{\partial}{\partial r}\left(r^{n} K(P) \frac{\partial P}{\partial r}\right)
$$

boundary conditions

\footnotetext{
*Corresponding author: VVVlasenko@nas.gov.ua
} 


$$
P\left(r_{0}, t\right)=f(t)+P_{S}, P(\infty, t)=P_{S}, f(0)=0
$$

where $f(t)$ - change pressure function at the boundary medium; $P_{S}$ - gas interstitial pressure in coal seam.

The function $f(t)$ describes both the injection mode and the pressure relief mode.

\section{Methods}

To solve the problem, the system of equations (1), (2) must be supplemented with a material breaking condition - a fracture criterion. The physical design of the destruction process reduce to next. As a result of pressure relief fluid is inversely filtered in the well, from the porous medium into it. In this case, the fluid pressure gradient in the volume adjacent to the well changes opposite sign. The gradient value is determined by the filtration characteristics of the fluid (viscosity, permeability coefficient), the injection and the velocity of its relief. The pressure gradient causes fluid movement in the pores. The transfer of a pulse, the flux density of which is equal to additional stresses in a condensed medium, takes place. Stresses are caused by impulse movement. Depending on the sign of the gradient, the force can be directed either toward free space (plane symmetry, $n=0$ ), wells (cylindrical symmetry, $n=1$ ), (spherical symmetry, $n=2$ ) or in the direction of the loading material. The stresses that cause the destruction of the material during pressure release are considered.

Let's consider some variants of plane, cylindrical and spherical symmetry successively. For all the variants, let's introduce the "force" criterion of destruction, the essence of which is going to next: destruction of the material occurs only if any surface tensile stresses exceed the material tensile strength

$$
\sigma_{i n} \geq \sigma_{b},
$$

where $\sigma_{i n}-$ tension intensity; $\sigma_{b}-$ tensile strength at break.

In the plane case, the acting force on an elementary volume of length $d r$ and a unit cross-sectional area $S_{0}$ is equal to

$$
S_{0}=(d P / d r) d r
$$

where $r$ - linear coordinate of selected volume.

In the quasistatic approximation, when it is possible to take the velocity propagation value of elastic perturbations equal to infinity, the force in the section with the coordinate $R$ is equal to

$$
F(R)=m \cdot S \int_{0}^{R} \frac{d P}{d r} d R=m \cdot S_{0}[P(R)-P(0)]
$$

where $m$ - the porosity of the substance (only the fluid part of the material participates in the creation of the stress state).

Substituting (5) into (3), the force criterion for destruction is obtained

$$
\sigma_{i n}(R)=\frac{F(R)}{S_{0}}=[P(R)-P(0)] \cdot m \geq \sigma_{b},
$$

From (6), it follows the stress curve corresponds to the dependence of the fluid pressure on the coordinate $R$ within the accuracy of scale factor $m$. In the case of cylindrical 
symmetry, the force acting on the elementary volume can be represented as:

$$
F(R)=l_{0} 2 \pi r^{2} \frac{\partial P}{\partial r} d r
$$

where $l_{0}$ - unit length of an elementary volume in a cylindrical coordinate system.

The resulting force acting on a radius cylindrical surface $R$ and a unit length $l_{0}$ is equal:

$$
F(R)=2 \pi l_{0} m \int_{r_{0}}^{R} r \frac{d P}{d r} d r=2 \pi l_{0} m\left[R \cdot P(R)-r_{0} P\left(r_{0}\right)-\int_{r_{0}}^{R} P(r) d r\right] .
$$

The corresponding expression for the force criterion is:

$$
\sigma_{i n}(R)=\frac{F(R)}{2 \pi R l_{0}}=m\left[P(r)-\frac{r_{0}}{R} P\left(r_{0}\right)-\frac{1}{R} \int_{r_{0}}^{R} P(r) d r\right] \geq \sigma_{b} .
$$

In the case of spherical symmetry, the force acting on the elementary volume can be represented as:

$$
F(R)=4 \pi r^{2} \frac{\partial P}{\partial r} d r
$$

The resulting force acting on a spherical surface of radius $R$ is equal to

$$
F(R)=4 \pi m \int_{r_{0}}^{R} r^{2} \frac{\partial P}{\partial r} d r=4 \pi m\left[R^{2} P(R)-r_{0}^{2} P\left(r_{0}\right)-2 \int_{r_{0}}^{R} r P(r) d r\right]
$$

Therefore

$$
\sigma_{i n}(R)=\frac{F(R)}{2 \pi R l_{0}}=m\left[P(R)-\frac{r_{0}^{2}}{R^{2}} P\left(r_{0}\right)-\frac{2}{R^{2}} \int_{r_{0}}^{R} r P(r) d r\right] \geq \sigma_{b} .
$$

Thus, if at some undetermined time $t_{\mathrm{j}}$ the momentum flow density in a certain section of the material exceeds the tensile strength, then the material will crack.

Material fissuring at HDI is possible only in the case when in a certain section both the force criterion [2] and the energy criterion are fulfilled. The main point of the energy criterion is as follows. During the inverse filtering, the forces of the HDI move the fluid in the pores

$$
A=m P u d t
$$

Taking into consideration the fact that $u=-k(\partial P / \partial r)$, a mathematical expression for the energy criterion of destruction is obtained

$$
-m \int_{0}^{t_{j}} k(P)\left(\frac{\partial P}{\partial r}\right) \cdot P d t \geq 2 g_{s},
$$

where $g_{s}$ - the formation energy density of the "fresh" surface.

The sign "-" in the right part of (14) is taken cause of the reason that only those volumes of matter we are interested, where $u<0(d P / d r>0)$. In this zone, the substance undergoes a 
tensile intensity from the side of the moving fluid. Destruction under the influence of compressive effects are not considered, because of the material stress limit in compression significantly exceeds the tensile strength [3]. Now therefore, the fulfillment of conditions (3) and (14) in a certain section of the medium means its destruction (spalling).

The formation of a free surface during the spallation drastically changes the conditions of the task, namely, at the breaking point $\left(r_{i}\right)$, the pressure should be equated to the pressure at the boundary of the fluid injection region. Therefore, further calculation should taking into account the conditions

$$
P\left(r_{i}, t\right)=P\left(r_{0}, t\right)
$$

The physical reason for this condition is the following: the transfer rate of elastic disturbance in a solid porous medium is very high (on the order of a kilometer per second) $[4,5]$. In view of this, the fluid pressure in the pores is "instantly" set equal to the pressure at the boundary of the impact area at the material fissure point during the formation of the free surface.

Thus, in the present formulation of the task real characteristics of the medium are taken into consideration:

- porosity of the material $m(P)$, depending on mining conditions;

- filtration coefficient $K(P)$;

- fluid viscosity;

- stressing area boundary size $r_{0}$;

- task symmetry (plane, cylindrical, spherical);

Characteristics of the HDI process:

- fluid injection mode is described by the function $f(t)$;

- impact time;

- pressure relief mode.

\section{Results and discussion}

Establishing the general patterns of HDI on a coal massif is pretty tricky task. The overcome both the mathematical difficulties caused by the backflip of the boundary conditions, the large volume of computational operations, and the multiparameters setting is necessary when it solving [6]. Accordingly, the establishment of particular patterns, knowledge of which is necessary for conducting experimental investigation and for improving the mathematical model.

Poisson's coefficient and Young's modulus $E$ are the most stable exemplify parameters for all types of coal. The remaining parameters are not obvious, except $P_{s}, \sigma_{b}, g_{s}, P_{2}$, which are currently considered the most significant in the process of a sudden blast.

Taking into consideration the complexity of the problem, it is most rational to calculate of HDI at parameter's values close to experimental investigation that was previously realized.

Based on the foregoing next task parameter values are set: $v=0.18 ; E=2.5 \cdot 10^{4} \mathrm{~Pa}$; $H=1000 \mathrm{~m} ; m_{\mathrm{s}}=0.05 ; P_{\mathrm{s}}=2 \cdot 10^{6} \mathrm{~Pa} ; k_{0}=4.4 \cdot 10^{-12} \mathrm{~m}^{2} /(\mathrm{Pa} \cdot \mathrm{s}) ; R_{1}=4.4 \cdot 10^{-19} \mathrm{~m}^{2} /\left(\mathrm{Pa}^{2} \cdot \mathrm{s}\right) ;$ $t_{2}=240 \mathrm{~s} ; P_{2}=10^{7} \mathrm{~Pa} ; P_{\mathrm{k}}=10^{5} \mathrm{~Pa} ; T_{\mathrm{c}}=0.1 \mathrm{~s} ; \sigma_{b}=1 \cdot 10^{6} \mathrm{~Pa} ; g_{s}=10 \mathrm{~Pa} \cdot \mathrm{m} ; R_{w}=0.075 \mathrm{~m}$.

Thus, the purpose of these calculations is to establish the role of each foregoing HDI process parameters, namely, on the process of destruction of the coal massif.

As practical experience shows [7, 8,9], the breaking strength of a coal substance has an essential role in initiation of a sudden blast process. Therefore, the role of this parameter in the HDI process is investigated.

In these calculations, the size of the computational domain was chosen $R_{N}=2 \mathrm{~m}$, the 
number of denumerable points $N=800$. All other parameters are equal to the foregoing. The time step in the fluid flow calculation was $0.5 \mathrm{step}$, taken at pressure relief. Trial calculations have shown that reducing this step does not affect the obtained results.

The variable parameter is the coal breaking strength, which, according to [10], is $\sim 10^{6} \mathrm{~Pa}$.

According to the calculations, the value $\sigma_{b}$ get properties of the critical value. If this parameter is less than a certain value, then the process of pressure relief and the following fluid (water) outflow is accompanied by the coal massif cracking, the final aim of the HDI is achieved. At the same time, gas emission in coal increases, and mechanical stresses decrease. If the coal breaking strength exceeds the critical, the HDI process ends with nothing, in the context of its practical application: as a result of the filtration process, the fluid injected into the well penetrates into the adjacent coal layers and flows into the well without generating any destruction of the coal mass during the following pressure relief.

With the foregoing parameters, the critical value of coal strength $\sigma_{c r}=3.1 \cdot 10^{6} \mathrm{~Pa}$. This means that if $\sigma_{b} \leq \sigma_{c r}$, realized the cracking process, the dynamics of which is shown in Figures $1-5$.

The top diagrams show the distribution of water pressure in the coal pores, depending on the distance to the well $P(R)$ at different moments. The middle graphs represent the same dependences for the energy density transferred to the coal material as a inverse filtration result of the liquid $E(R)$.
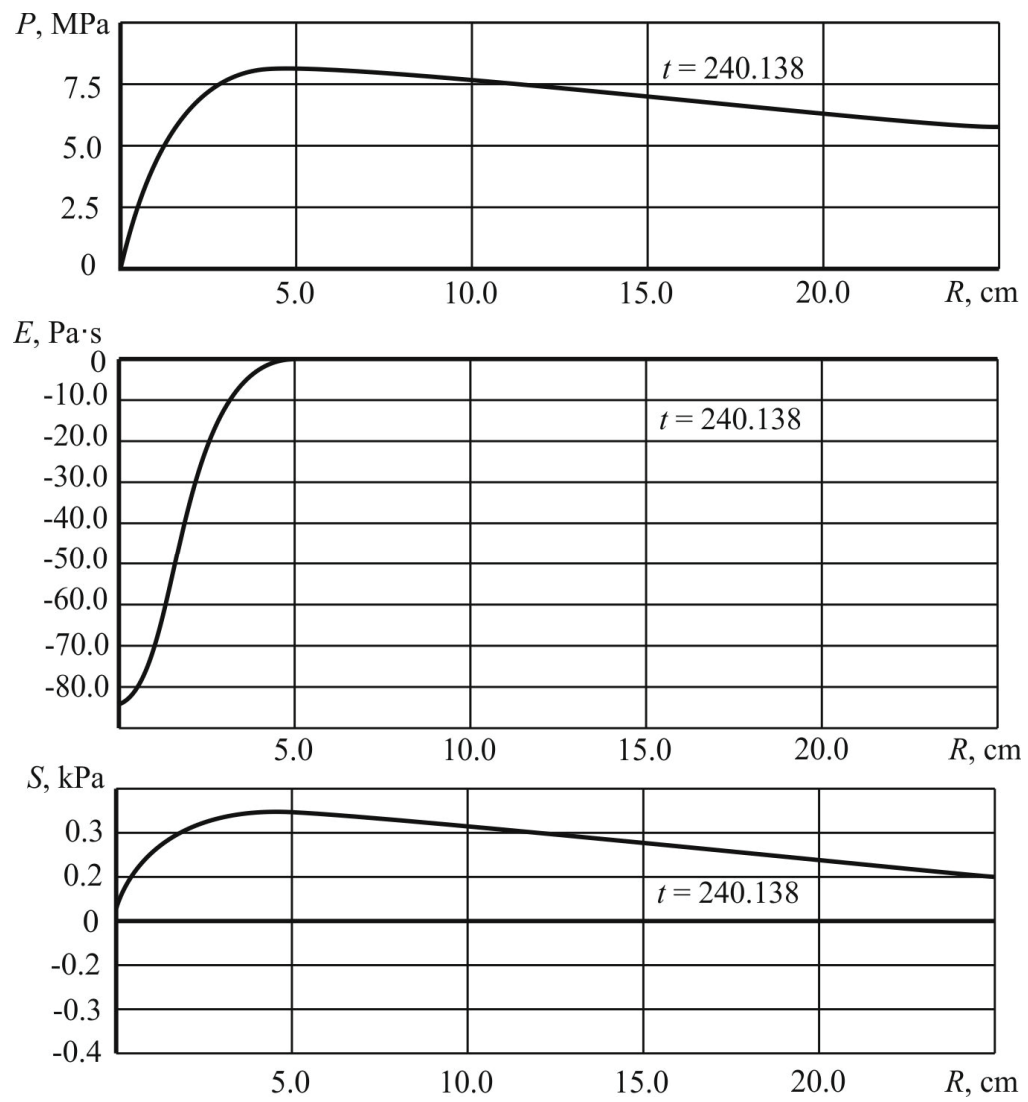

Fig. 1. Main settings of HDI spreading at the moment of time before the formation of a new crack. 

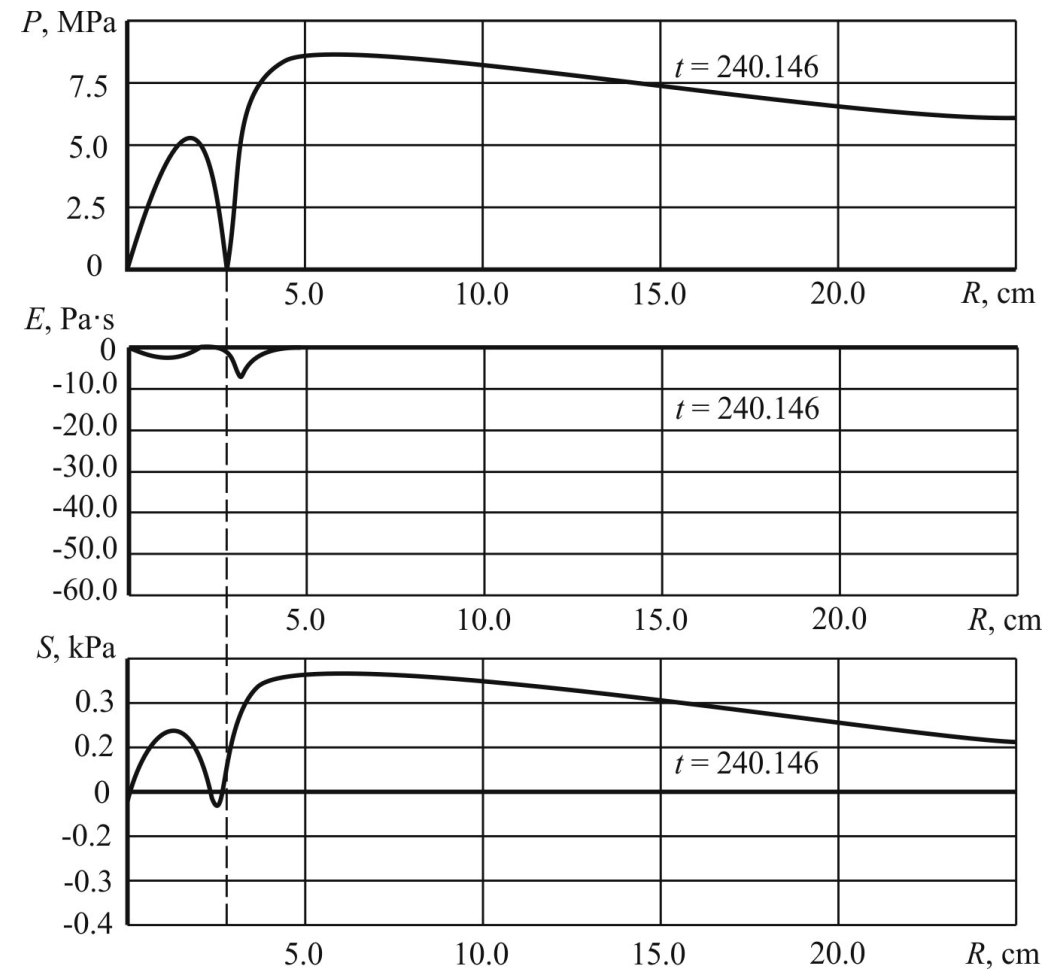

Fig. 2. Main settings of HDI spreading at the moment of time before the formation of a second crack.
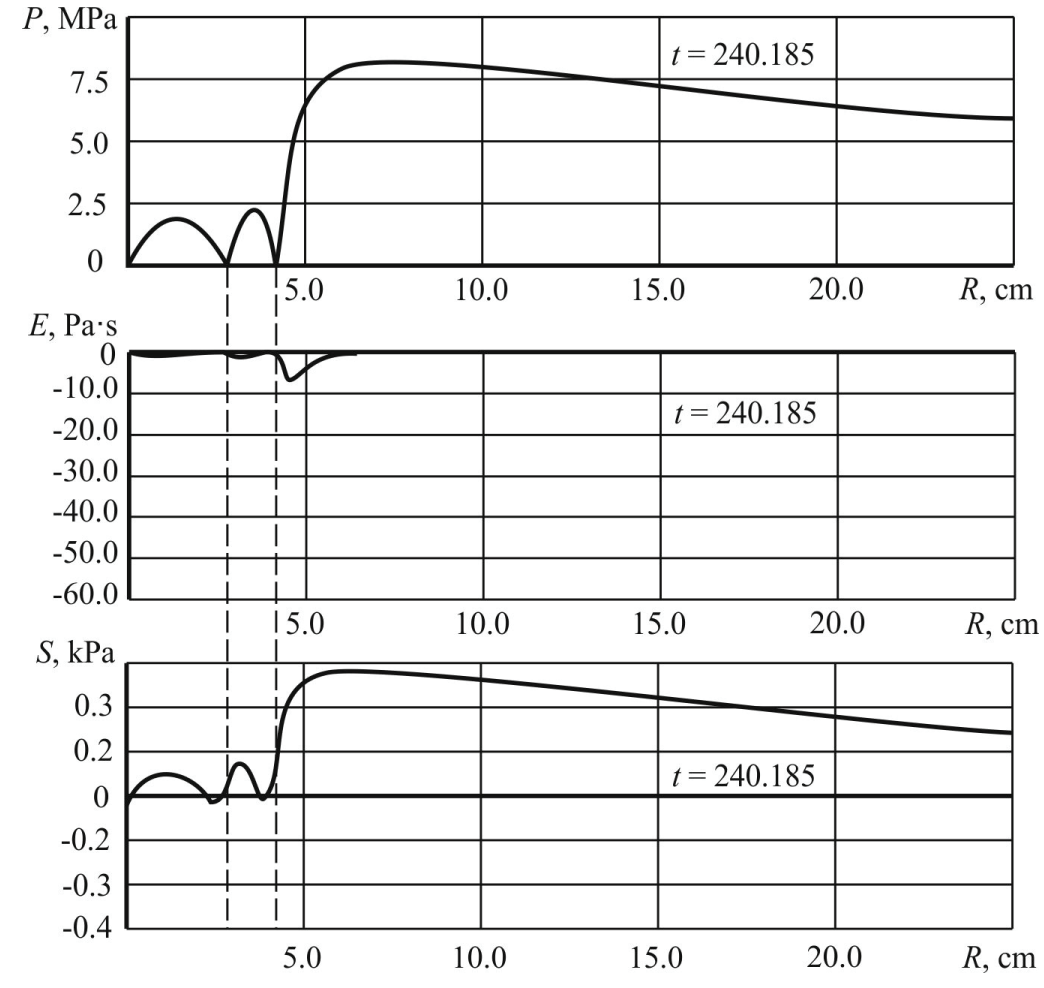

Fig. 3. Main settings of HDI spreading at the moment of time after the formation of a second crack. 


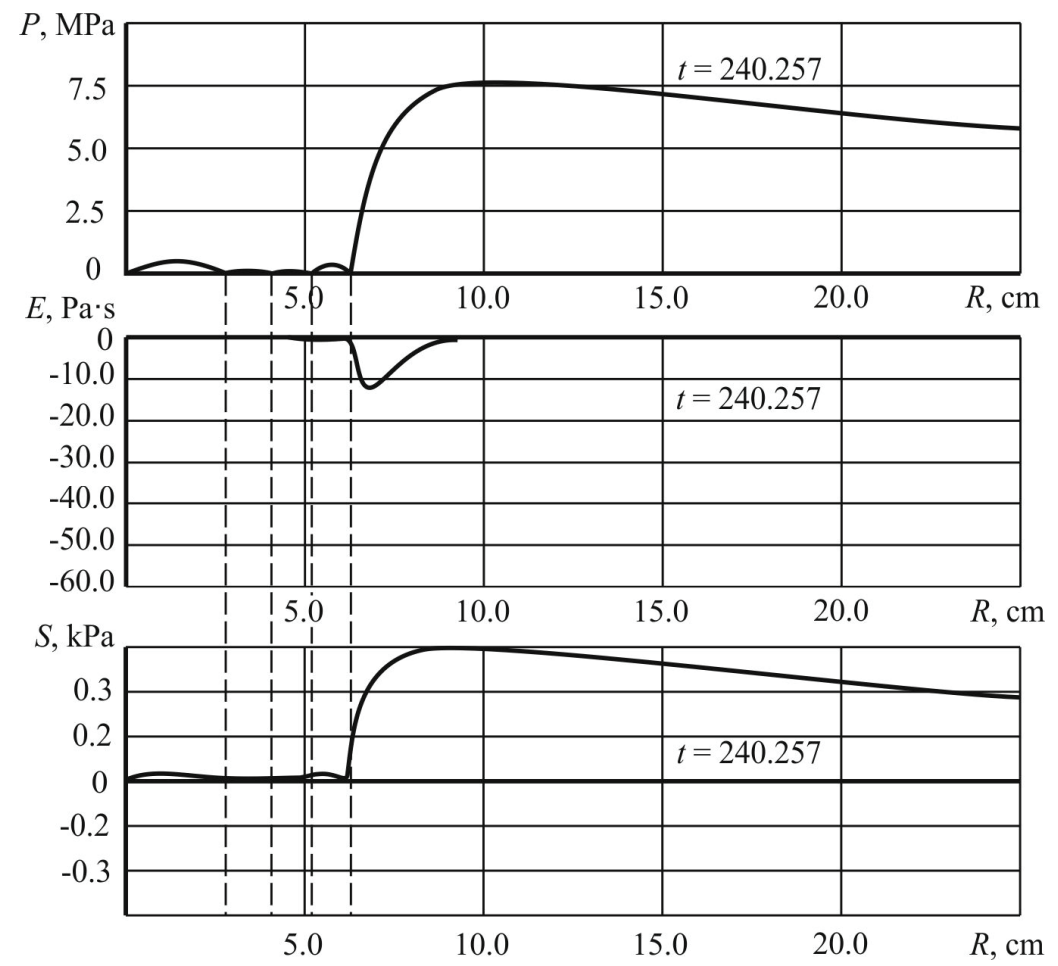

Fig. 4. Future spreading main settings of HDI.
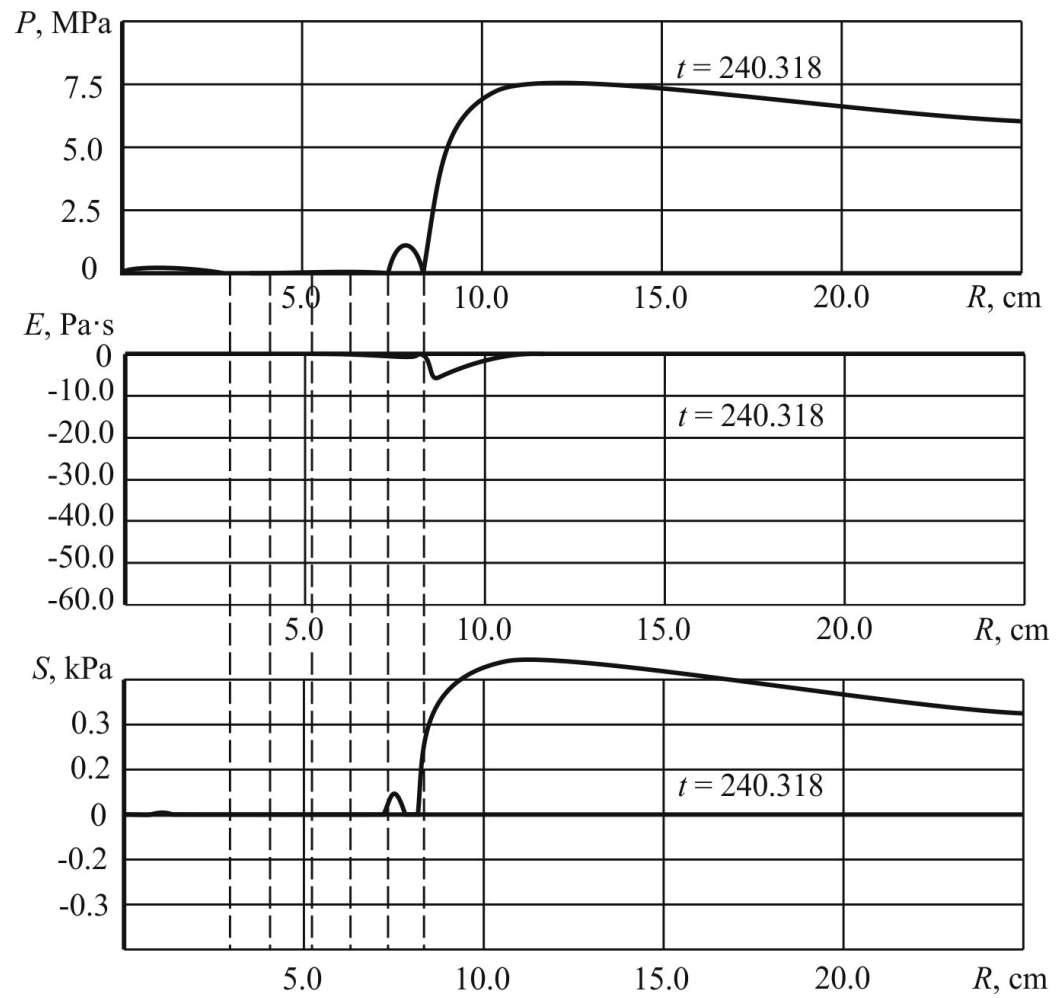

Fig. 5. Main settings of HDI spreading when entering stationary mode of the cracking process. 
Negative values of this energy indicate that the fluid flows in the direction opposite to the direction of the radius vector. The lower graphs are dependences of stresses in the coal coming out from water filtration $S(R)$. Vertical lines intersecting the middle and lower graphs indicate the formation of cracks in coal.

The distribution of the main HDI parameters at the time moment that precedes the formation of the first crack is shown in Figure 1. In spite of the fact that the coal material, accumulates internal energy, considerably exceeding the energy of formation of a "fresh" surface, as a result of the inverse filtration $g_{s}=10 \mathrm{~Pa} \cdot \mathrm{m}$, the rupture of a substance does not occur cause of the destruction force criterion is failed. In other quarters, the process of the cracks formation requires the fulfillment of two criteria: the power and energy at the same time on any cylindrical surface of the substance. This is corresponding to a point in time. $t=240.138 \mathrm{~s}$. Figure 2 is corresponding to a point in time $t=240.146 \mathrm{~s}$, when the formation of the first crack began and the formation of the second is going. It can be seen that the maximum of dependencies $E(R)$ и $S(R)$ in space do not match and are separated by distance $\sim 3 \mathrm{~cm}$. Therefore, the conditions for the formation of cracks at this point in time have not been created yet, despite the fact that the maximum values of $E$ and $S$ exceed the critical values $g_{c r}=10 \mathrm{~Pa} \cdot \mathrm{m}, \sigma_{c r}=3.1 \cdot 10^{6} \mathrm{~Pa}$. Figure 3 shows the distribution $P(R), E(R), S(R)$ after the formation of the second crack. It is seen that the second piece of coal formed is smaller in size than the first piece.

Further process dynamics is shown in Figure 4 and Figure 5. Moreover, Figure 4 corresponds time to the stationary mode of the cracking process, which will continue until the water-gas boundary is reached.

\section{Conclusions}

During the HDI on a coal massif, it is possible to influence on the stress state by the changing the fluid flow mode in the pore space. To determine the process parameters that can vary filtering modes, a physical model of the process and an algorithm for its mathematical solution was developed.

1. In other quarters, the process of the cracks formation requires the fulfillment of two criteria: the power and energy at the same time on any cylindrical surface of the substance.

2. With a decrease of the strength of the coal substance on the crack, the generation of the first crack is carried out earlier than in the above calculations; the size of the first piece of coal decreases with decreasing $\sigma_{b}$ relative to the value $\sigma_{\text {cr }}$ in relation to the value; the distances between the cracks are equidistant; the water pressure in the pores of the coal formed pieces quickly tends to the pressure value in the well.

\section{References}

1. Samaskiy, A.A. (1989). Teoriia raznostnyh shem. Moskva: Nauka

2. Matchenko, V.A., Sadovenko, I.A. (2014). Doslidzhennya osoblivosti deformatsii neidealno-pruzhnih girskih porid, Naukonyy visnyk NMU, (6), 80-86

3. Sofiyskiy, K.K., Silin, D.P., Vlasenko, V.V., Kolchin, G.I. (2012). Izmeneniye sostoyaniya gornogo massiva $\mathrm{v}$ rezultate gidrodinamicheskogo vozdeystviya, Materials deformation and destruction with defects and dynamic phenomena in rocks and working, 317-319

4. Sadovenko, I.A., Inkin, A.V., Yakubovskaya, Z.N., Maksimova-Gulyayeva, N.A. (2012). Otsenka poter gaza pri yego khranenii v vodonosnykh plastakh Zapadnogo Donbassa, Naukovyy visnyk NMU, (6), 18-24

5. Sadovenko, I.O., Inkin, O.V., Zholudev, S.V. (2012). Mihratsiya i teploperenos 
navkolo pidzemnoho hazoheneratora, Dnipropetrovsk: National Mining University

6. Zorin, A.N., Kolesnikov, V.G., Didenko, A.T. (1984). Impulsno-volnovoye vozmushcheniye $\mathrm{v}$ skvazhine pri pnevmogidravlicheskom sposobe dobychi uglya, Fiziko-tekhnicheskiye problemy razrabotki poleznykh iskopayemykh, (4), 88-92

7. V. Vlasenko, V. Gavrilov, O. Moskovskiy, Influence of hydrodynamic impact on degassing of steep coal seam at the top of the overhead longwall, New developments in mining engineering Theoretical and practical solutions of mineral resolurces mining, CRC Press/Balkema: EN Leiden, The Netherlands (2015)

8. V. Vlasenko, R. Agaiev, K. Dudlia, M. Kyrychenko, D. Prytula, On the Possibility of Coalbed Methane Extraction as a Source of Energy under the Hydrodynamic Impact on the Outburst Coal Seam, Advanced Engineering Forum Submitted, Switzerland: Trans Tech Publications, 25, 2017

9. R. Agaiev, O. Katulskyi, D. Prytula, Intensification of coalbed methane recovery through the surface degasing borehole, New developments in mining engineering Theoretical and practical solutions of mineral resolurces mining, CRC Press/Balkema: EN Leiden, The Netherlands, (2015)

10. Vlasenko, V.V., Zhitlenok, D.M., Moskovskiy, O.V. Starodubtsev, S.V. (2009). Kriteriy razrusheniya uglya pri gidrodinamicheskom vozdeystvii na plast, Geotekhnicheskaya mekhanika [Geo-technical mechanics], (81), 227-232 